\title{
The Spectral Cell Method in Nonlinear Earthquake Modeling
}

\author{
Daniel Giraldo ${ }^{1}$ and Doriam Restrepo ${ }^{2}$
}

\begin{abstract}
In this study we focus at examining an efficient high order finite element strategy to compute the dynamic response of heterogeneous basins under nonlinear soil behavior subjected to point-source earthquake events. The numerical technique known as the Spectral Cell Method (SCM) combines Fictitious-Domains concepts with the Spectral-version of the finite element method to accurately solve the wave equations in heterogeneous geophysical domains. We tested the SCM in physically and computationally challenging domains namely, (i) a semi-elliptical basin, and (ii) an undulated basin embedded in a half-space with several irregular geological structures with different material discontinuities. Nonlinear behavior is considered by implementing a Mohr-Coulomb, and a DruckerPrager yielding criteria. We benchmark our simulations with results obtained using MIDAS GTS NX, a finite element tool for geotechnical applications based upon traditional boundary-fitted meshing techniques.
\end{abstract}

\section{Introduction}

In the last decades seismologist have become increasingly aware of the importance of high-performance numerical methods to accurately predict the dynamic response of complex geological domains. Among the adopted strategies, the finite difference method and the octree version of finite element method have dominated the bulk of numerical seismic studies of large sedimentary basins (e.g., Frankel, 1993; Olsen and Archuleta, 1996; Bao et al., 1998; Bielak et al., 2005; Moczo et al., 2007; Graves and Pitarka, 2010; Taborda and Bielak, 2013). High-order finite element methods in the form of the spectral finite element method have also emerged as accurate tools to perform seismic wave propagation simulations at global, regional, and continental scales with notable accuracy and high-order convergence rates (e.g., Komatitsch and Vilotte, 1998; Komatitsch and Tromp, 2002a,b; Stupazzini et al., 2009; Cupillard et al., 2012). In addition to their intrinsic differences low and high order techniques significantly differ in their adopted meshing strategies. Meshing in the finite difference method and the low-order finite element method rely on structured/un-structured meshes of regular elements to accommodate internal geometries of realistic S-wave structures. Neither strictly conform with internal material discontinuities to solve the elastic wave equations, consequently, accuracy is decreased at the expense of low discretization effort. In contrast, the improved accuracy of the spectral element method comes in part at the price of significant computational burden due to the restriction of high-quality boundary-conforming hexahedral meshes, which renders a considerable amount of time in the case of realistic geologic volumes (Casarotti et al., 2008; Cupillard et al., 2012).

\footnotetext{
${ }^{1}$ Graduate Student. Universidad EAFIT. E-mail: dgiral32@eafit.edu.co

${ }^{2}$ Civil Engineering Department. Associate Professor. Universidad EAFIT. Carrera 49 No 7 Sur - 50. Medellín - Colombia. Tel.: +57 (4) 261 9337; E-mail: drestre6@eafit.edu.co
} 
In full contrast with traditional domain decomposition strategies, the Finite Cell Method (FCM) (Parvizian et al., 2007; Düster et al., 2008) combines fictitious domain ideas (Saul'ev, 1963) with highorder hierarchical finite elements P-FEM (Szabo and Babuška, 1991) to approximate the solution of partially differential equations in difficult domains. Since the method uses cartesian grids the meshing process is greatly simplified. The FCM implicitly considers the geometry of the domain at the integration stage of the procedure. Adopting quad-, and oc-tree recursive divisions for adaptive integration in 2D and 3D applications plus a weakly imposition of boundary conditions, the FCM has shown to maintain the exponential rates of convergence in the energy norm known from the $P$-FEM (Parvizian et al., 2007). Important applications of the FCM have been successfully reported in areas ranging from geometrically non-linear continuum mechanics (e.g., Schillinger et al., 2012), biomedical engineering (e.g., Ruess et al., 2012), and elastoplasticity (e.g., Abedian et al., 2013). In dynamic problems on the other hand, the FCM exhibits fully populated mass matrices. This, in conjunction with the lack of an appropriate mass lumping technique precludes the applicability of the method in explicit dynamics. The Spectral Cell Method (SCM) introduced by Duczek et al. (2014a) circumvents this shortcoming by changing from hierarchical Legendre basis to Lagrange node-interpolation functions, and by adopting coincident interpolation nodes with quadrature point locations, i.e., optimal lumping (Cook et al., 1989). Areas of applicability of the SCM have been reported in ultrasonic guided wave propagation analysis (Duczek et al., 2014a), wave propagation in heterogenous materials (e.g., Joulaian et al., 2014), and multiphysics applications (e.g., Zander et al., 2012; Duczek et al., 2014b).

In the context of earthquake ground motion analysis on the other hand, to the best knowledge of the authors, the SCM has not being explored so far. The latter might be explained by the satisfactory results obtained in traditional linear earthquake modeling. However, even when low magnitude events might drive a soil response mostly linear, it is now widely accepted that large magnitude earthquakes are responsible of triggering important nonlinear soil response (Field et al., 1998). Despite this recognized behavior, the inclusion of the nonlinear soil response in the numerical analysis of seismic induced ground motion is still reserved to sensitive engineering projects. Due to the complexity of advanced soil constitutive models and to the computational challenges arising in their implementation, practitioner engineers and seismologists still rely upon equivalent linear models (Seed and Idriss, 1969) to quantify the extent of nonlinear behavior in their simulations. The salient features of spectral strategies might help to alleviate such shortcomings. It is well known that Lagrange node-interpolation shape functions exhibit superior features than classical $h$-finite elements as its shape functions closely resemble terms in a Fourier series, what leads to high convergence rates, and larger element sizes(Joulaian et al., 2014). Theoretically, the method only requires $G=\pi$ points per wavelength in order to accurately simulate the propagation of seismic waves with zero occurrence of numerical dispersion (Ainsworth and Wajid, 2009). In practical situations Seriani and Priolo (1994) suggested that $G=4.5$ is needed for $P=8$ polynomial order, which still allows the use of very coarse meshes. As a result, spectral concepts accurately generate elemental stiffness matrices as needed when dealing with highly heterogeneous velocity gradients as well as provide an accurate numerical integration framework of complex constitutive soil models essential in geoplasticity analysis.

This paper aims at examining the benefits of the SCM in assessing the dynamic response of heterogeneous basins under elastic and non-linear soil conditions subjected to hypothetical point-source earthquake events. We present a set of simulations in domains ranging from ideal alluvial basins as in Gelagoti et al. (2012), to irregular geological settings with several material discontinuities. As previously mentioned, soil nonlinearity has been traditionally accounted for through the use of equivalent linear models deeply rooted in the engineering practice (Field et al., 1998). In this work we adopt concepts of geoplasticity that offer a sound framework for formulating specific features of soil behavior. In particular we implemented a Mohr-Coulomb and a Drucker-Prager yielding criteria as constitutive relations. Other authors have employed these soil models in their studies of nonlinear seismic wave propagation (e.g., 
Xu et al., 2003; Restrepo et al., 2011; Taborda et al., 2012). Both models assume elasto-plastic behavior of the soil deposits in conjunction with non-associative plastic flow rules. We present comparisons of accuracy and convergence with benchmark solutions obtained using MIDAS GTS NX (Midas-GTS-NX, 2016), a finite element tool for geotechnical applications based on boundary-fitted meshes.

\section{The Spectral Cell Method (SCM)}

Assume a heterogeneous body $\Omega$ of traction-free boundary $\Gamma=\Gamma_{N} \cup \Gamma_{D} ; \Gamma_{N} \cap \Gamma_{D}=\emptyset$, where $\Gamma_{N}$ and $\Gamma_{\mathrm{D}}$ denote Newmann and Dirichlet boundaries, subjected to body forces represented by the vector $\mathbf{f}_{\mathbf{b}}$. In seismic wave propagation studies $\mathbf{f}_{\mathbf{b}}$ represents the rupture characteristics of the seismic source. Writing down the weak form of the elastodynamic equation for zero initial conditions one obtains the well known expression:

$$
\int_{\Omega} \rho(\mathbf{x}) \mathbf{v} \cdot \ddot{\mathbf{u}} d \Omega+\int_{\Omega} \boldsymbol{\varepsilon}(\mathbf{v}): \sigma(\mathbf{u}) d \Omega=\int_{\Omega} \mathbf{v} \cdot \mathbf{f}_{\mathbf{b}} d \Omega,
$$

where $\rho(\mathbf{x})$ is the density function, $\boldsymbol{\varepsilon}$ and $\sigma$ are the strain and stress tensors respectively, while $\mathbf{u}$ and $\mathbf{v}$ are the displacement and test functions respectively.

The discrete solution of eq. (1) traditionally calls for a mesh made of conforming elements capable of aligning to any geometric irregularity of the domain, and that follows every material discontinuity within the region of study. In contrast, the SCM being in essence a Fictitious Domain strategy, reduces its meshing requirements to two simple stages. First, the target domain $\Omega$ is embedded into a larger, simpler, domain $\tilde{\Omega} \backslash \Omega$ of homogeneus Dirichlet $\tilde{\Gamma}_{\mathrm{D}}$ and Newmann $\tilde{\Gamma}_{\mathrm{N}}$ boundaries. Displacements and tractions are assumed continuous across the original interface $\Gamma_{\mathrm{N}}$. Since the material exceeds the physical boundaries its effect is minimized by the material penalization parameter $\alpha(\mathbf{x})$. Rewritten eq. (1) for the expanded domain one obtains:

$$
\int_{\tilde{\Omega}} \alpha(\mathbf{x}) \rho(\mathbf{x}) \mathbf{v} \cdot \ddot{\mathbf{u}} d \tilde{\Omega}+\int_{\tilde{\Omega}} \alpha(\mathbf{x}) \boldsymbol{\varepsilon}(\mathbf{v}): \sigma(\mathbf{u}) d \tilde{\Omega}=\int_{\tilde{\Omega}} \alpha(\mathbf{x}) \mathbf{v} \cdot \mathbf{f}_{\mathbf{b}} d \tilde{\Omega},
$$

where:

$$
\alpha(\mathbf{x})=\left\{\begin{array}{cl}
1, & \text { if } \mathbf{x} \in \Omega \\
10^{-q}, & \text { if } \mathbf{x} \notin \Omega .
\end{array}\right.
$$

For the limiting case $q=\infty$ eq. (2) reduces to the exact formulation as in traditional finite element analysis. In practice $q$ is a problem dependent variable although $5 \leq q \leq 10$ has been successfully reported as a proper range to avoid numerical instabilities (Joulaian et al., 2014). In the earthquake modeling context, the most prevalent region of study consists of traction-free domains idealized as rectangular areas (box-shaped volumes in 3D) capable of including large sedimentary basins and the geometry of the rupture zones. As a result, there is no need to extend the domain beyond its physical boundaries. The external boundaries $\tilde{\Gamma}$ and $\Gamma$ coincide, and naturally reduce to four orthogonal lines (six orthogonal planes in 3D). On the other hand, $\Omega$ is usually composed of several sub-domains $\Omega_{i}$, each of whom representing the interior geological units. Since the domain is free of voids or empty spaces, the material penalization function is always $\alpha(\mathbf{x})=1$. We noted however that $\alpha(\mathbf{x})$ turns into an indicator function used to identify the parent material of queried points across material interfaces. Even more, in this study $\alpha(\mathbf{x})$ is also used to implicitly pinpoint those sub-regions where nonlinear behavior will be allowed.

In the second stage of the method, the increased domain is discretized by means of a "Cartesian" grid. The ensuing regular, non-overlapping square elements are denoted as "Cells" to state the fact 
that these do not align with the boundaries of $\Omega$ as their finite element counterparts. In practice, cells completely outside $\Gamma$ are removed in order to avoid ill-conditioned matrices. Figure 1. depicts a 2D domain as illustrative example.
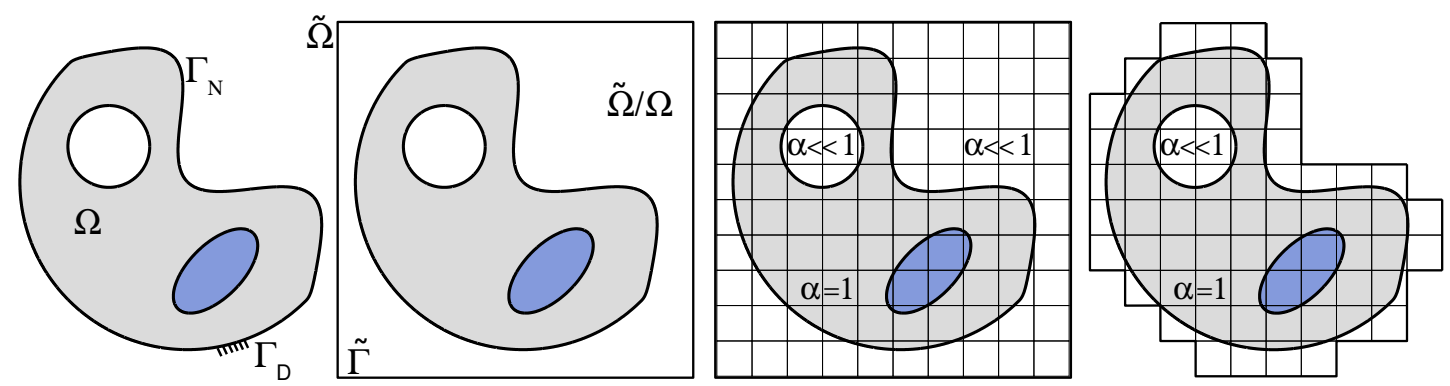

Figure 1: The physical domain $\Omega$ is embeded in the fictitious domain $\tilde{\Omega}$, which can be solved using a regular grid. Here, $\alpha(\mathbf{x})$ penalizes elements cut by the void.

Following standard Bubnov-Galerkin ideas, the displacement and test functions are approximated according to:

$$
\mathbf{u}=\mathbf{N U} ; \mathbf{v}=\mathbf{N V}
$$

where $\mathbf{N}$ is the array of approximated shape functions, and $\mathbf{U}$ and $\mathbf{V}$ denote the nodal values of the displacement and test functions. Plugging in eq. (4) into eq. (2) and introducing the damping matrix $\mathbf{C}$ one obtains:

$$
\mathbf{M U}+\mathbf{C} \dot{U}+F_{\text {Int }}=\mathbf{F},
$$

where:

$$
\mathbf{M}=\int_{\tilde{\Omega}} \alpha(\mathbf{x}) \rho(\mathbf{x}) \mathbf{N}^{\top} \mathbf{N} d \tilde{\Omega}, \quad \mathbf{F}_{\mathbf{I n t}}=\int_{\tilde{\Omega}} \alpha(\mathbf{x}) \mathbf{B}^{\top} \sigma d \tilde{\Omega}, \quad \mathbf{F}=\int_{\tilde{\Omega}} \alpha(\mathbf{x}) \mathbf{N}^{\top} \mathbf{f}_{\mathbf{b}} d \tilde{\Omega} .
$$

The matrix $\mathbf{M}$ represents the mass of the system, while $\mathbf{C}$ denotes the material attenuation usually accounted for through the material quality factor $Q$. If linear material behavior is assumed, another form of attenuation can be expressed as a linear combination between the mass and stiffness matrices. Although no intrinsic damping was considered in this study, the presence of Lysmer conditions (Lysmer and Kuhlemeyer, 1969) along every external boundary of the domain but the free surface brings forth the damping matrix into our formulation.

The terms $\mathbf{B}$ and $\mathbf{F}_{\text {Int }}$ denote the kinematic strain matrix and the vector of internal nodal forces respectively. This representation of the internal material response allows us to satisfy every particular characteristic of the nonlinear material model in use. Under linear behavior the material response reduces to the standard form $\mathbf{F}_{\text {Int }}=\mathbf{K U}$ expressed in terms of the linear stiffness matrix $\mathbf{K}$, which is obtained from the contribution of each cell stiffness matrix $\mathbf{k}_{c}$. For cells of order $P$ the elemental stiffness matrix is a $(p+1) \times(p+1)$ block symmetric matrix. The $(i, j)_{\text {th }}$ term is a $(2 \times 2)$ sub-block matrix of the form:

$$
\begin{gathered}
\mathbf{k}_{\mathbf{c}(i, j)}=(\lambda+\mu)\left[\begin{array}{cc}
a_{i j} & c_{i j} \\
c_{i j} & b_{i j}
\end{array}\right]+\mu\left(a_{i j}+b_{i j}\right)\left[\begin{array}{ll}
1 & 1 \\
1 & 1
\end{array}\right] \\
a_{i j}=\int_{-1}^{+1} \int_{-1}^{+1} \frac{\partial N_{i}}{\partial \xi} \frac{\partial N_{j}}{\partial \xi} d \xi d \eta ; \quad b_{i j}=\int_{-1}^{+1} \int_{-1}^{+1} \frac{\partial N_{i}}{\partial \eta} \frac{\partial N_{j}}{\partial \eta} d \xi d \eta ; \quad c_{i j}=\int_{-1}^{+1} \int_{-1}^{+1} \frac{\partial N_{i}}{\partial \xi} \frac{\partial N_{j}}{\partial \eta} d \xi d \eta,
\end{gathered}
$$


where $\lambda$ and $\mu$ are the linear material constants. $N_{i}(\xi, \eta)$ is the $i_{\text {th }}$ shape function expressed in terms of Lagrange polynomials $L_{m}(\xi)$ in the interval $[-1,1]$ :

$$
N_{i}(\xi, \eta)=L_{m}(\xi) L_{n}(\eta), \quad L_{m}(\xi)=\prod_{\substack{j=1 \\ j \neq m}}^{p+1} \frac{\xi-\xi_{j}}{\xi_{m}-\xi_{j}}, \quad i=(p+1)(m-1)+n,
$$

the nodes spatial distribution $\xi$ is defined by the roots of the Lobatto polynomials. This distribution is commonly known as the Gauss-Lobatto-Legendre (GLL) grid.

\subsection{Numerical Integration}

Since the SCM uses non-conforming Cartesian grids the meshing process is greatly reduced. The computational price for such a simplification is however shifted to the numerical integration of eqn 6 and 7 . If the cell is cut by different materials, a quadtree adaptive strategy is the preferred scheme to numerically cope with discontinuous integrands, and to implicitly comply with the real boundaries of the domain (e.g, Parvizian et al., 2007; Ruess et al., 2012). This is particularly true in nonlinear sub-regions where the correct identification of material interfaces is paramount. The quadtree scheme recursively divide the cell into four lower-level subcells until either the subcell is filled with one single material, or the quadtree has reached the maximum level of sub-division.

If the subcell is either fully immersed into a nonlinear region, or it is partially filled with more than one material, its internal force contribution is numerically obtained by standard Gauss point integration schemes. Interpolation functions of order $P$ will require $P+1$ Gauss points per side in order to ensure integration accuracy. Such a requirement will however populate the parent cell with quadrature points, which will severely affect the computational efficiency of the method specially if nonlinear behavior is expected. Here we successively reduce the Gauss quadrature rule as the quadtree goes its way up to the maximum level of sub-division. Other authors have reduced the quadrature rule down to one single Gauss point (e.g., Abedian et al., 2013) based on the premise that interpolation functions could be considered as lower-order polynomials in the lowest level subcells. Here, we set the minimum number of Gauss points to two per side in order to reduce the effect of staggered material interfaces (see fig. 2).
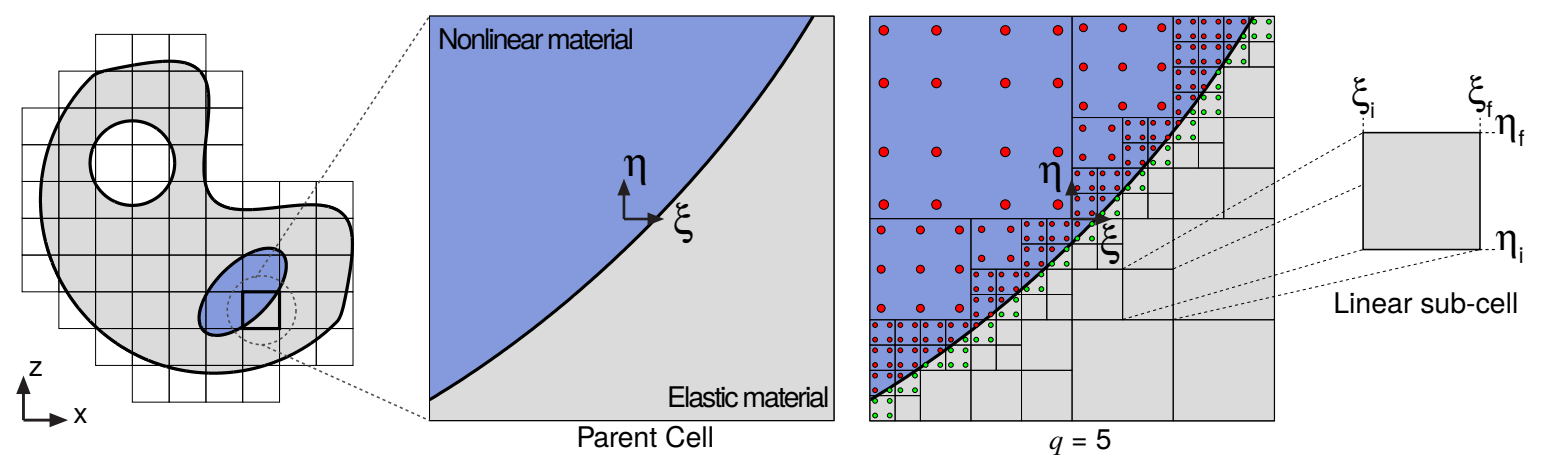

Figure 2: Adaptive integration using $q=5$ decomposition levels. The red and green quadrature points correspond to the elasto-plastic material and the elastic material respectively. The elemental stiffness matrix is calculated with the contribution of the subcells completely immersed into the elastic material and the evaluation of the green points. The red points are used to compute the non-linear portion of the total potential force.

On the other hand, the contribution of linear subcells to the internal force vector is computed analytically after re-writing $L_{m}(\xi)$ in eq. (8) as: 


$$
L_{m}(\xi)=a_{0_{m}}+a_{1_{m}} \xi+a_{2_{m}} \xi^{2}+\ldots+a_{p_{m}} \xi^{p} \quad m=1,2,3 \ldots \ldots . p+1 .
$$

Using the zero-one property of the Legendre polynomials in conjunction with the positions $\xi_{p}$ of the GLL grid, one can easily show that the coefficients of the $P+1$ polynomials in eq. (9) correspond to the terms of the inverse of $\mathbf{A}$ :

$$
\mathbf{A}=\left(\begin{array}{ccccc}
1 & \xi_{0} & \xi_{0}^{2} & \cdots & \xi_{0}^{p} \\
1 & \xi_{1} & \xi_{1}^{2} & \cdots & \xi_{1}^{p} \\
1 & \xi_{2} & \xi_{2}^{2} & \cdots & \xi_{2}^{p} \\
\vdots & \vdots & \vdots & \ddots & \vdots \\
1 & \xi_{p} & \xi_{p}^{2} & \cdots & \xi_{p}^{p}
\end{array}\right) .
$$

In other words, the $b_{i j}$ term of $\mathbf{A}^{-1}$ is the $i_{\text {th }}$ coefficient of the $j_{\text {th }}$ Legendre polynomial of order $P$. With this scheme we explicitly compute the products of gradients shown in eq. (7) and analytically solve the prescribed integrals over the subcell interval $\xi_{\mathrm{i}} \leq \xi \leq \xi_{\mathrm{f}}, \eta_{\mathrm{i}} \leq \eta \leq \eta_{\mathrm{f}}$ (see fig. 2). In comparison with the numerical integration, we noted that the analytic alternative reduces the preprocessing computational time for quadtree levels $q \leq 5$ and polynomials degrees up to $P=5$.

\subsection{Mass Lumping}

Explicit dynamics heavily depends on lumped mass strategies to compute the evolution in time of the dynamic response. This requirement is naturally achieved by SEM as long as the quadrature points coincide with a GLL distribution. Since cells of homogeneous density fulfill this condition, their elemental mass matrices will be diagonal by construction. Mass matrices of cells exhibiting different densities could as well be treated following a GLL quadrature. We however adopt as lumping alternative the HRZ technique (Hinton et al., 1976). This method has been effectively used in the analysis of cells exhibiting voids and/or holes (e.g., Joulaian et al., 2014; Duczek et al., 2014c). The HRZ strategy builds the lumped mass matrix $\mathbf{m}_{\mathrm{c}}$ after preserving the total mass of the cell $m_{\text {cell }}$, and the principal diagonal of the consistent mass matrix $\mathbf{m}_{\mathrm{c}}^{\mathrm{cst}}$.

$$
\mathbf{m}_{\mathrm{c}}^{\mathrm{cst}}=\int_{\Omega_{c}} \alpha(\mathbf{x}) \rho(\mathbf{x}) \mathbf{N}^{T} \mathbf{N} d \Omega_{c}, \quad m_{\mathrm{cell}}=\int_{\Omega_{c}} \alpha(\mathbf{x}) \rho(\mathbf{x}) d \Omega_{c}, \quad \mathbf{m}_{\mathrm{c}}=\frac{2 m_{\mathrm{cell}}}{\operatorname{Tr}\left(\mathbf{m}_{\mathrm{c}}^{\mathrm{cst}}\right)} \operatorname{diag}\left(\mathbf{m}_{\mathrm{c}}^{\mathrm{cst}}\right) .
$$

All the integrals are computed using adaptive integration. Due to the latter, the computational cost increases with respect to the GLL quadrature. The method however is expected to have improved accuracy due to its capacity to track the spatial distribution of mass within the cell.

\section{Nonlinear formulation}

Under consideration of small strains, we apply the classical additive decomposition of the total strain tensor $\varepsilon=\varepsilon^{e}+\varepsilon^{p}$ in the context of rate-independent plasticity. The stress tensor follows the linear relation $\sigma=\mathbf{C}:\left(\varepsilon-\varepsilon^{p}\right)$, where $\mathbf{C}$ is the fourth-order tensor of elastic moduli. In order to set the moment when the plasticity takes place, classical plasticity defines a yielding criterion and a plastic flow rule. Both of them in conjunction with loading/unloading and consistency conditions establish the set of admissible stresses. The yielding criterion and plastic flow are defined respectively as follow:

$$
f(\boldsymbol{\sigma}, \boldsymbol{\beta}) \leq 0, \quad \dot{\boldsymbol{\varepsilon}}^{p}=\gamma \frac{\partial g}{\partial \boldsymbol{\sigma}},
$$


where $\beta$ is the hardening parameters vector and $\gamma$ is so-called plastic multiplier. Since we assume a non-associative flow rule and perfect plasticity conditions, the plastic potential $g$ and the yield function $f$ differ and $\boldsymbol{\beta}=\mathbf{0}$. Regarding the constitutive model, there are several realistic yielding criteria to predict the nonlinear soil behaviour such as the Modified Cam-Clay (Roscoe and Burland, 1968). This model for instance is suitable to describe the behaviour of consolidated soft soils. In the same vein, Hoek and Brown (1980) proposed a model to characterize the strength of jointed rock masses. Sekiguchi and Ohta (1977) implemented a model capables to predict the nature of anisotropically consolidated clays. We focus on the Mohr-Coulomb and Drucker-Pager models (Drucker and Prager, 1952) because these are the ones mostly used in geotechnical engineering projects.

The Mohr-Coulomb yielding criterion states that yielding occurs when the shearing stress and normal stress reach a linear combination

$$
\tau=c-\sigma \tan (\phi),
$$

where $\phi$ is the angle of friction and $c$ is the cohesion of the material. This equation can be rewritten as yielding function

$$
f(\sigma, \phi, c)=\left(\sigma_{\text {Max }}-\sigma_{\text {Min }}\right)+\left(\sigma_{\text {Max }}+\sigma_{\text {Min }}\right) \sin (\phi)-2 c \cos (\phi) \leq 0 .
$$

Here, $\sigma_{\text {Max }}$ and $\sigma_{\text {Min }}$ are the maximum and minimum principal stress respectively. On the other hand, the Drucker-Prager model corresponds to a smoothed version of the Mohr-Coulomb criterion that removes the edges and corners of the hexagonal pyramid. This leads to computational reductions in the material update process. The yielding function is defined as

$$
f(\sigma, \phi, c)=\sqrt{J_{2}(\sigma)}+a \cdot p(\sigma)-b \cdot c \leq 0, \quad a=\frac{2 \sqrt{3} \sin (\phi)}{3-\sin (\phi)}, \quad b=\frac{2 \sqrt{3} \cos (\phi)}{3-\sin (\phi)},
$$

where $J_{2}(\sigma)$ is the second invariant of the deviatoric stress tensor, $p(\sigma)$ is the hydrostatic stress and $c$ is the cohesion. The values of $a$ and $b$ make the Drucker-Prager criterion concident at the outer edges of the Mohr-Coulomb criterion. For both models, the function $g$ in the plastic flow rule is determine by evaluating $f$ in the dilatance angle $\psi<\phi$, i.e., $g=f(\sigma, \psi, c)$. The material update process is carried out using the well known elastic-predictor/plastic-corrector scheme or Return-Mapping Algorithm (Simo and Hughes, 1998; de Souza Neto et al., 2008).

\section{Numerical Examples}

The ability of the SCM is validated by carrying out two numerical experiments. Namely, a semi-elliptical basin with three impedance contrast with respect to the bedrock, and an undulated basin embedded in a irregular geological setting. To validate the results obtained by the SCM, we use MIDAS GTS-NX, a finite element analysis software package widely used by practitioner engineers. The cells size are defined according to the meshing rule:

$$
\Delta h=\frac{V_{s_{\min }}}{P P W \cdot f_{\max } \cdot P},
$$

where $V_{s_{\min }}$ is the minimum S-wave velocity of the domain, $f_{\max }$ is the maximum frequency in the simulation, $P P W$ are the points per wavelength and $P$ is the polynomial degree of the interpolation functions. 


\subsection{Semi-elliptical basin}

The shape of the basin is a semi-ellipse of semi-major axis $a=500 \mathrm{~m}$ and semi-minor axis $b=300$ $\mathrm{m}$. This basin is situated at the top of a half-space of constant P-wave velocity $V_{p}=1732 \mathrm{~m} / \mathrm{s}$, Swave velocity $V_{s}=1000$ and density $\rho=1800 \mathrm{Kg} / \mathrm{m}^{3}$. The validation process is carried out using different levels of impedance contrast $(I C)$, i.e., the ratio between the S-wave velocities of the basin and the half space. The basin exhibits a Mohr-Coulomb nonlinear behavior. In the first numerical experiment, denoted basin 0 , the velocities of the basin and the half-space coincide, therefore the impedance contrast $I C=1$. For the second experiment, $\operatorname{basin} 1$, the velocity of the basin is $V_{s}=750$ $\mathrm{m} / \mathrm{s}(I C=750 / 100=0.75)$. In the final experiment, the value of the S-wave velocity is $V s=500 \mathrm{~m} / \mathrm{s}$, i.e., $I C=0.5$. The seismic source is an isotropic point-source located at $(x, z)=(532.5,432.5)$ varying in time as a Ricker pulse of central frecuency $f_{c}=4 \mathrm{~Hz}$. The fig. 3 depicts the general features of the geometry, while table 1 shows the material properties of the simulations.

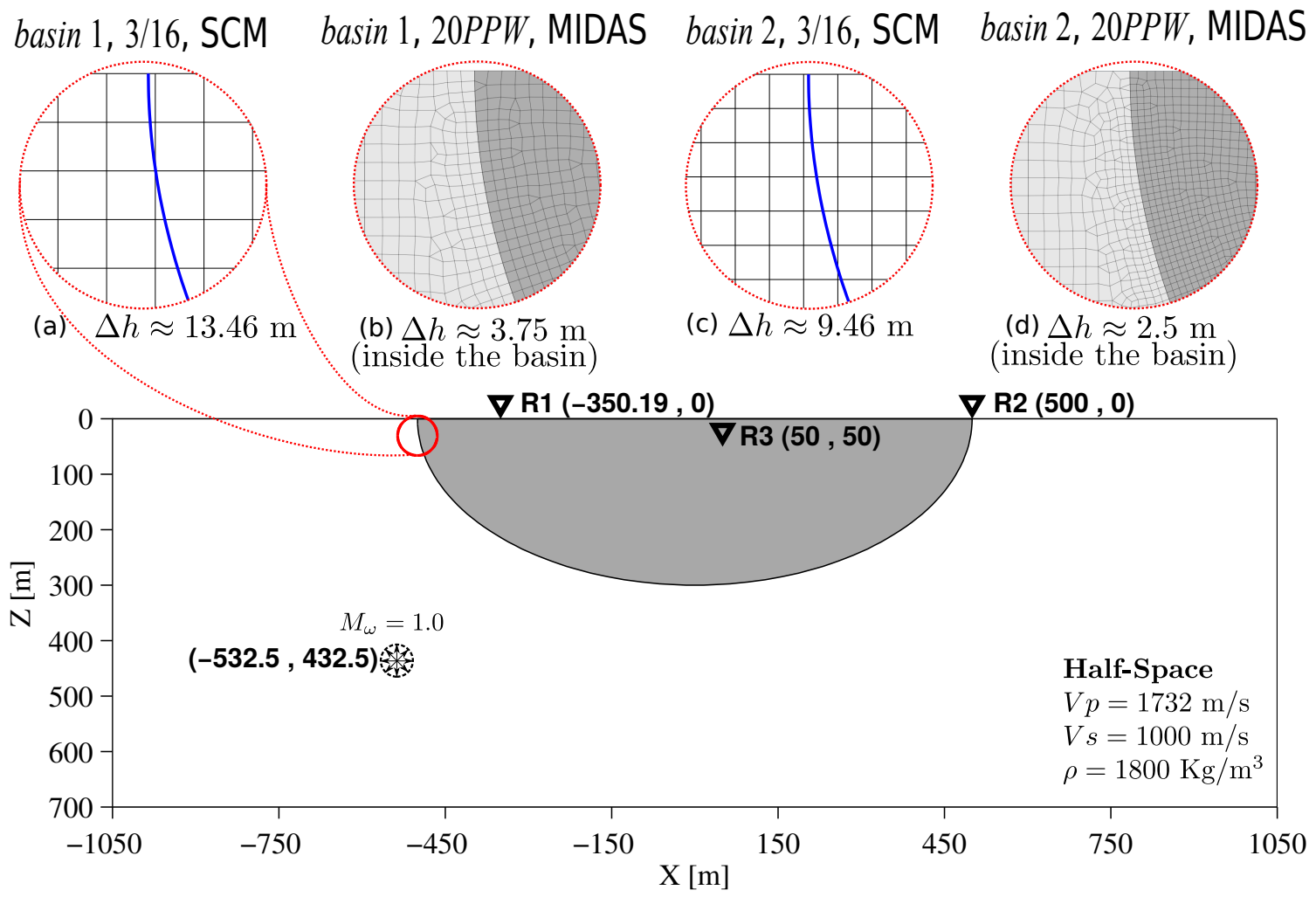

Figure 3: Cartesian and conforming meshes, general geometry, and properties of the semi-elliptical basin. Fig.3 (a) illustrates an enlarged view of the regular grid at the top right-hand corner of the semi-elliptical domain for simulation basin 1 . The parameter $3 / 16$ denotes $P=3$ and $P P W=16$ used in simulation basin 1 . Figure $3(\mathrm{~b})$ shows the mesh of the reference solution for the simulation basin 1 . The Figure 3(c) and 3(d) are the regular mesh of the SCM and the reference solution respectively for the simulation basin 2 . The points $\mathbf{R} \mathbf{1}, \mathbf{R} 2$ and $\mathbf{R} 3$ are the receivers whose results are analyzed.

The fig. 4 shows the comparison among results, for the horizontal and vertical displacement (left side) and velocity (rigth side) of the receivers R1, R2 and R3. These results correspond to $P / P W W$ combinations of $1 / 24$ for basin 0 and $3 / 16$ for basin 1 . Peaks and permanent deformations due to non-linear behavior of the basin are well captured by the SCM in comparison with the reference solution. The strong phase of the ground motion generates a significant irreversible deformation at receiver R1, but the SCM approach is capable of tracing this behavior. At station $\mathbf{R} 3$ of simulation basin 1, the displacement has a small desviation after $t=1 \mathrm{~s}$, but the record 


\begin{tabular}{|c|c|c|c|c|c|c|}
\hline Properties $\rightarrow$ & $V p(\mathrm{~m} / \mathrm{s})$ & $V s(\mathrm{~m} / \mathrm{s})$ & $\rho\left(\mathrm{Kg} / \mathrm{m}^{3}\right)$ & $c(\mathrm{Kpa})$ & $\phi\left(^{\circ}\right)$ & $\psi\left({ }^{\circ}\right)$ \\
\cline { 1 - 7 } Simulation $\downarrow$ & 1732 & 1000 & 1800 & 220 & 30 & 10 \\
\hline basin 0 & 1299 & 750 & 1800 & 190 & 30 & 10 \\
\hline basin 1 & 866 & 500 & 1800 & 160 & 30 & 10 \\
\hline basin 2 & &
\end{tabular}

Table 1: Material properties of the semi-elliptical basin simulations. The parameters $c, \phi$ and $\psi$ are the cohesion, frictional angle and dilatancy angle respectively.

shape is preserved. The velocity results on the other hand, show a good adjustment with respect to the reference solution for both simulations.

Figure 5 shows the quadrature points that reach the plastic limit. The three simulations used a $P / P P W$ combination of $1 / 24$ for basin $0,3 / 16$ for basin 1 , and $5 / 12$ for basin 2 . Note that most of quadrature points near the seismic source in the three simulations reach the plastic limit. Although a small region of plastic points appears in the bottom left side (region $\mathbf{A}$ in fig. 5) of the SCM results of basin 0 , the overall plasticity pattern is very similar for all simulations.

basin 0: Displacement [mm]

basin 0: Velocity $[\mathrm{cm} / \mathrm{s}]$

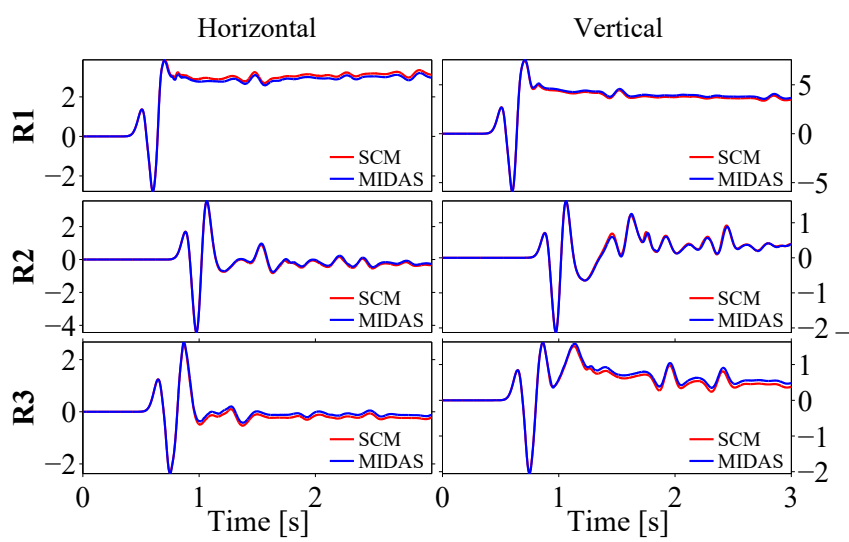

basin 1: Displacement [mm]

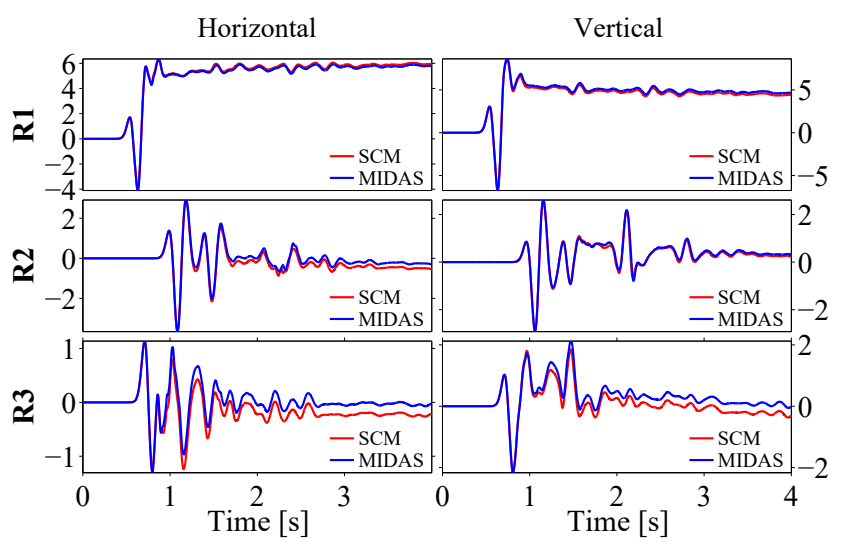

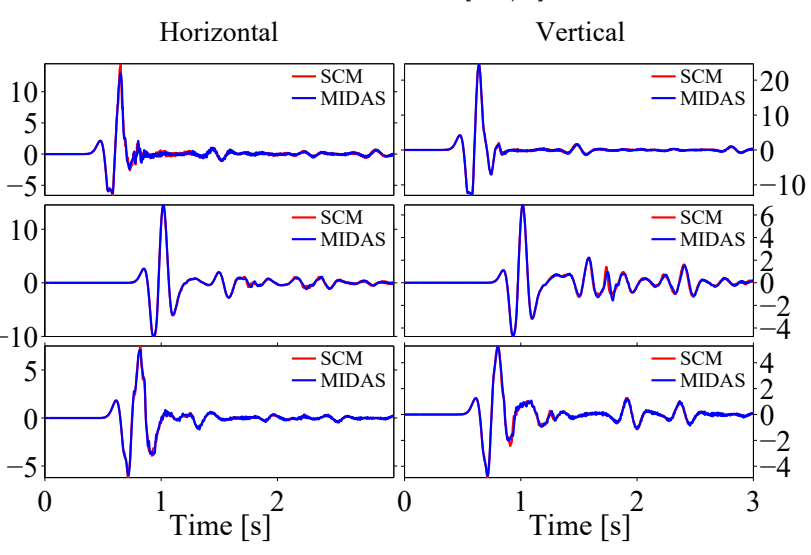

basin 1: Velocity $[\mathrm{cm} / \mathrm{s}]$

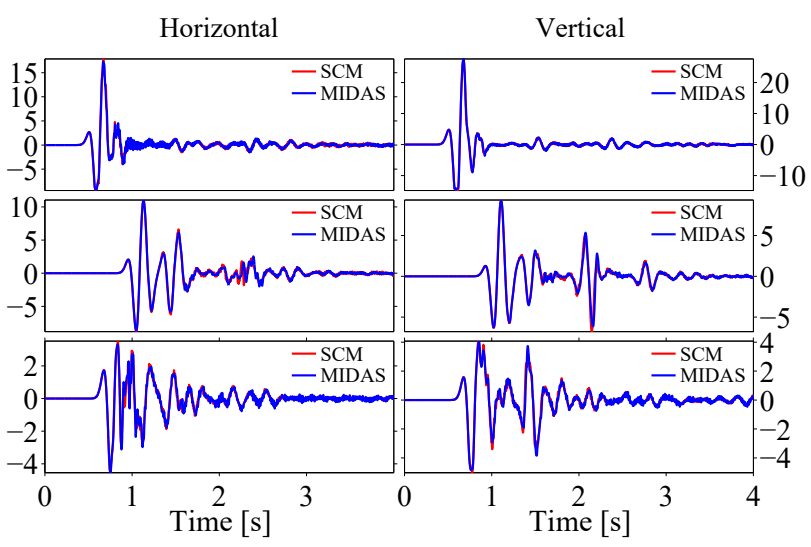

Figure 4: Comparison of the horizontal and vertical displacement $(\mathrm{mm})$ and velocity $(\mathrm{cm} / \mathrm{s})$ in basin 0 and basin 1 using a $P / P P W$ combination of $3 / 12$. The first three rows show the results of basin 0 evaluated at the three receivers. The last three rows correspond to basin 1 . The colum one and two are the horizontal and vertical displacements respectively. The column three is the horizontal velocity and the last one is the vertical velocity. The red line in each graph is the solution obtained by the SCM, while the blue line is the reference solution. 


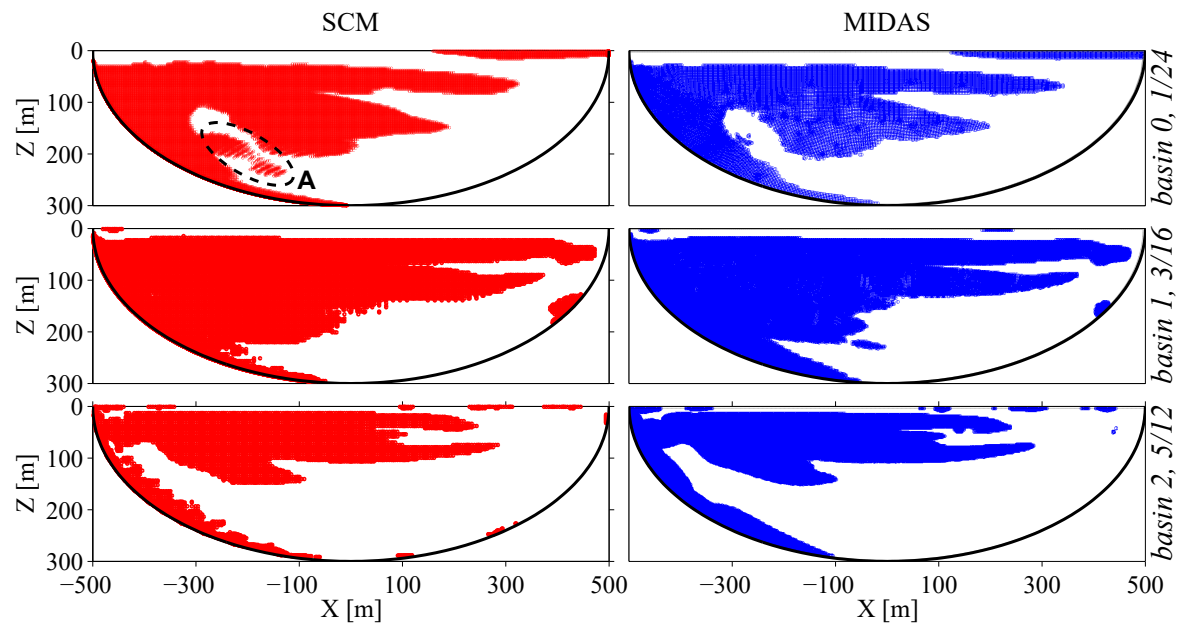

Figure 5: Zones of plastic activity. The first row corresponds to simulation basin 0 for a $P / P P W$ combination of $1 / 24$. The second row is the simulation basin 1 for $P / P W W=3 / 16$. The last row is the simulation basin 2 where the $P / P P W$ combination is $5 / 12$.

Spatial error maps are showing in fig. 6. To calculate the displacements error, we used the root mean square (rms) error proposed in Pelties et al. (2010):

$$
E_{k, i}^{u}=\sqrt{\frac{\int\left|U_{k, i}-R_{k, i}^{u}\right|^{2} d t}{\left.\int\left|R_{k, i}^{u}\right|\right|^{2} d t}} \approx \sqrt{\frac{\sum_{n=0}^{N}\left|U_{k, i, n}-R_{k, i, n}^{u}\right|^{2} \Delta t}{\sum_{n=0}^{N}\left|R_{k, i, n}^{u}\right|^{2} \Delta t}},
$$

where $U_{k, i, n}$ and $R_{k, i, n}^{u}$ are the displacement of the SCM and the reference solution respectively in the direction $k$ (horizontal or vertical) of the receiver $i$ at the time $\operatorname{step} n$. The velocity error measure is determined by the relative error of the Peak Ground Velocity (PGV) as follow:

$$
E_{k, i}^{v}=\frac{\left|\operatorname{PGV}\left(V_{k, i}\right)-\operatorname{PGV}\left(R_{k, i}^{v}\right)\right|}{\left|\operatorname{PGV}\left(R_{k, i}^{v}\right)\right|}, \quad \operatorname{PGV}\left(V_{k, i}\right)=\max _{n}\left|V_{k, i, n}\right|,
$$

where $V_{k, i, n}$ and $R_{k, i, n}^{v}$ are the velocity results of the SCM and the reference solution respectively. Figure 6 presents the error maps for the simulation basin 2. There is a reduction in the error measure from $54 \%$ to $37 \%$ approximately in the estimation of both displacements when the polynomial degree changes from $P=1$ to $P=3$ in the upper right part of the basin. However, the results of $P=3$ and $P=5$ show a similar distribution of the error inside the basin. For both, the error ranges between $7 \%$ and $50 \%$ approximately. On the other hand, the simulations with $P P W=16$ reduce the error from $26 \%$ to $16 \%$ approximately for the horizontal component with respect to $P P W=12$ at the middle of the basin. If we compared the results when $P P W=16$ and $P P W=24$, there is not an significant improvement.

Figure 6 (lower part) correspond to the relative error of the velocity field using the PGV measure. It is important to point out that the combination $1 / 16$ has the lowest error among all simulations schemes with horizontal and vertical errors of about $10 \%$ and 5\% respectively. The others schemes do not have an important change between them. Although their relative errors are lower than $15 \%$ for both components of motion.

Finally, table 2 exhibits the CPU time (serial programming), degrees of freedom (DoF) and sampling time $(\Delta t)$ of each scheme for simulation basin 2. Note that simulations with the same $P P W$ preserve approximately the number of degrees of freedom, although the CPU time exhibits a mixed behavior. It decreases from $P=1$ to $P=3$, but increases at $P=5$. This situation occurs because of the sampling time decreasing, and the subcell size increasing produced by the increment of the polynomial degree. Consequently, the decomposition level grows in order to perform the adaptive integral in the material interface. Accordingly, the number of quadrature points grows up in the cut elements therefore, the material update process takes a longer time. 

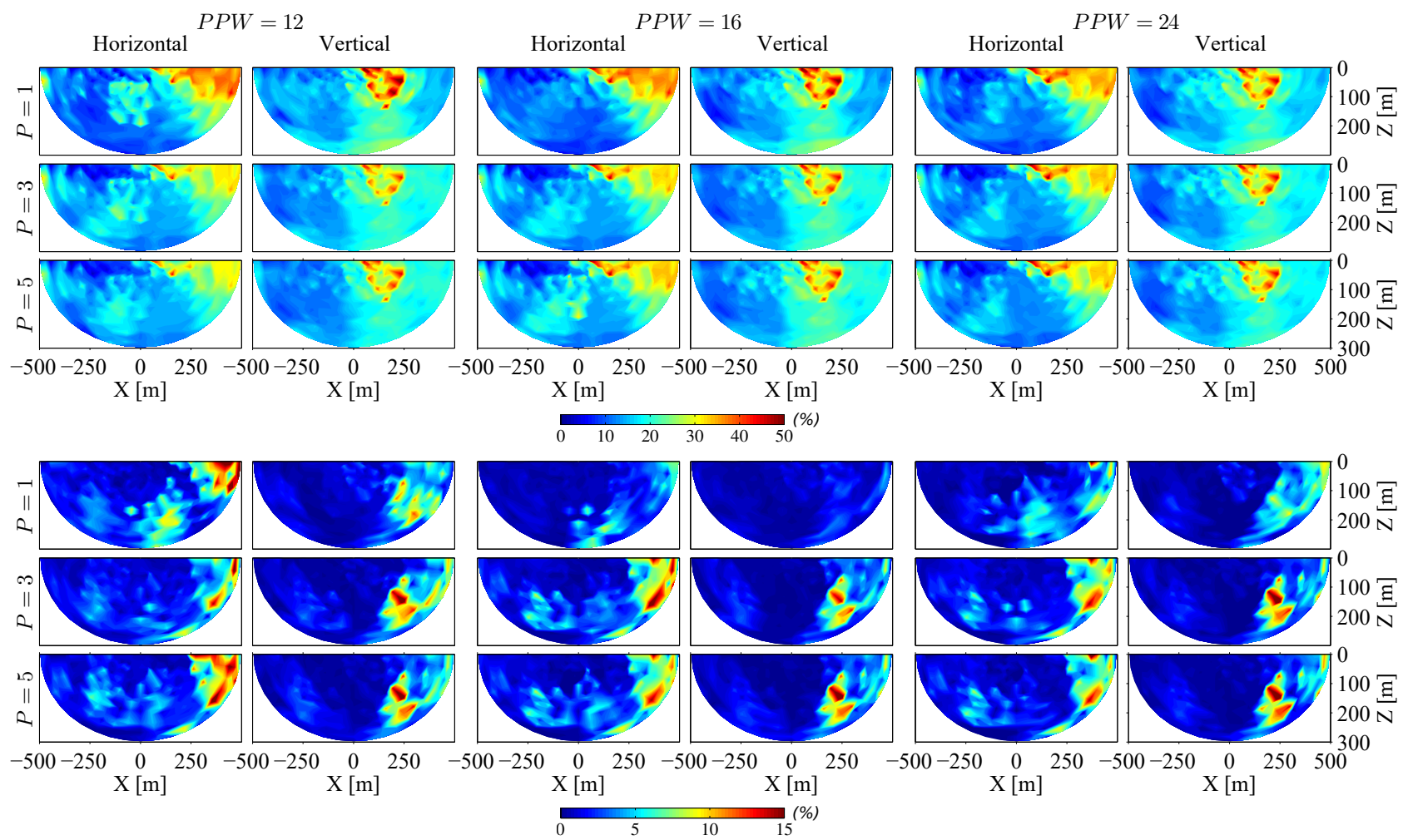

Figure 6: Error maps of simulation basin 2 inside the elasto-plastic region. Rows 1 to 3 and 4 to 6 correspond to the displacement and velocity error respectively. Here, it shows the error maps for all combinations $P / P P W$ of this experiment.

\begin{tabular}{|c|c|c|c|c|c|c|c|c|c|}
\hline $\begin{array}{c}\text { Points per } \\
\text { Wavelength }\end{array}$ & \multicolumn{3}{|c|}{$P P W=12$} & \multicolumn{3}{c|}{$P P W=16$} & \multicolumn{3}{c|}{$P P W=24$} \\
\hline $\begin{array}{c}\text { Polynomial } \\
\text { Degree }\end{array}$ & CPU Time & DoF & $\Delta t(\mathrm{~s})$ & CPU Time & DoF & $\Delta t(\mathrm{~s})$ & CPU Time & DoF & $\Delta t(\mathrm{~s})$ \\
\hline$P=1$ & $\begin{array}{c}6 \text { hours } \\
43 \text { mins }\end{array}$ & 170690 & $7.22 \times 10^{-4}$ & $\begin{array}{c}13 \text { hours } \\
52 \text { mins }\end{array}$ & 302850 & $5.41 \times 10^{-4}$ & $\begin{array}{c}43 \text { hours } \\
9 \text { mins }\end{array}$ & 680066 & $3.61 \times 10^{-4}$ \\
\hline$P=3$ & $\begin{array}{c}5 \text { hours } \\
26 \text { mins }\end{array}$ & 170690 & $5.98 \times 10^{-4}$ & $\begin{array}{c}11 \text { hours } \\
15 \text { mins }\end{array}$ & 297482 & $4.53 \times 10^{-4}$ & $\begin{array}{c}31 \text { hours } \\
25 \text { mins }\end{array}$ & 680066 & $2.99 \times 10^{-4}$ \\
\hline$P=5$ & $\begin{array}{c}12 \text { hours } \\
52 \text { mins }\end{array}$ & 174762 & $4.19 \times 10^{-4}$ & $\begin{array}{c}20 \text { hours } \\
22 \text { mins }\end{array}$ & 292162 & $3.24 \times 10^{-4}$ & $\begin{array}{c}64 \text { hours } \\
39 \text { mins }\end{array}$ & 696322 & $2.09 \times 10^{-4}$ \\
\hline
\end{tabular}

Table 2: CPU time, degrees of freedom and sampling time of all schemes in simulation basin 2.

\subsection{Irregular geological setting}

The domain is a half-space with six different geological structures where two of them exhibit a non-linear behavior (see fig. 7). Regions 1 to 4 have elastic properties. Regions 5 and 6 have elasto-plastic properties according to the Mohr-Coulomb and the Drucker-Prager yield criteria respectively. The material properties of the regions are described in table 3.

The validation process is carried out using $P / P P W$ combinations of $1 / 12,1 / 16,3 / 12$ and $3 / 16$. The element size $\Delta h$ is calculated according to eq. (16), where $V_{S_{\min }}$ is the S-wave velocity of region 6 . Five receivers are located at critical points of the domain (see fig. 7). The seismic source is an isotropic point-source located at $(x, z)=(-540,640)$ with time-varying function as a Ricker pulse of central frequency $f_{c}=4 \mathrm{~Hz}$.

Figure 8 shows the time evolution of plasticity areas inside the elasto-plastic regions. Note that for all time steps, the SCM follows with high resolution the history of the plasticity. On the other hand, fig. 9 shows the displacement and velocity fields of the five receivers (see fig. 7) for $P / P W W=3 / 12$. The SCM correctly predicts 


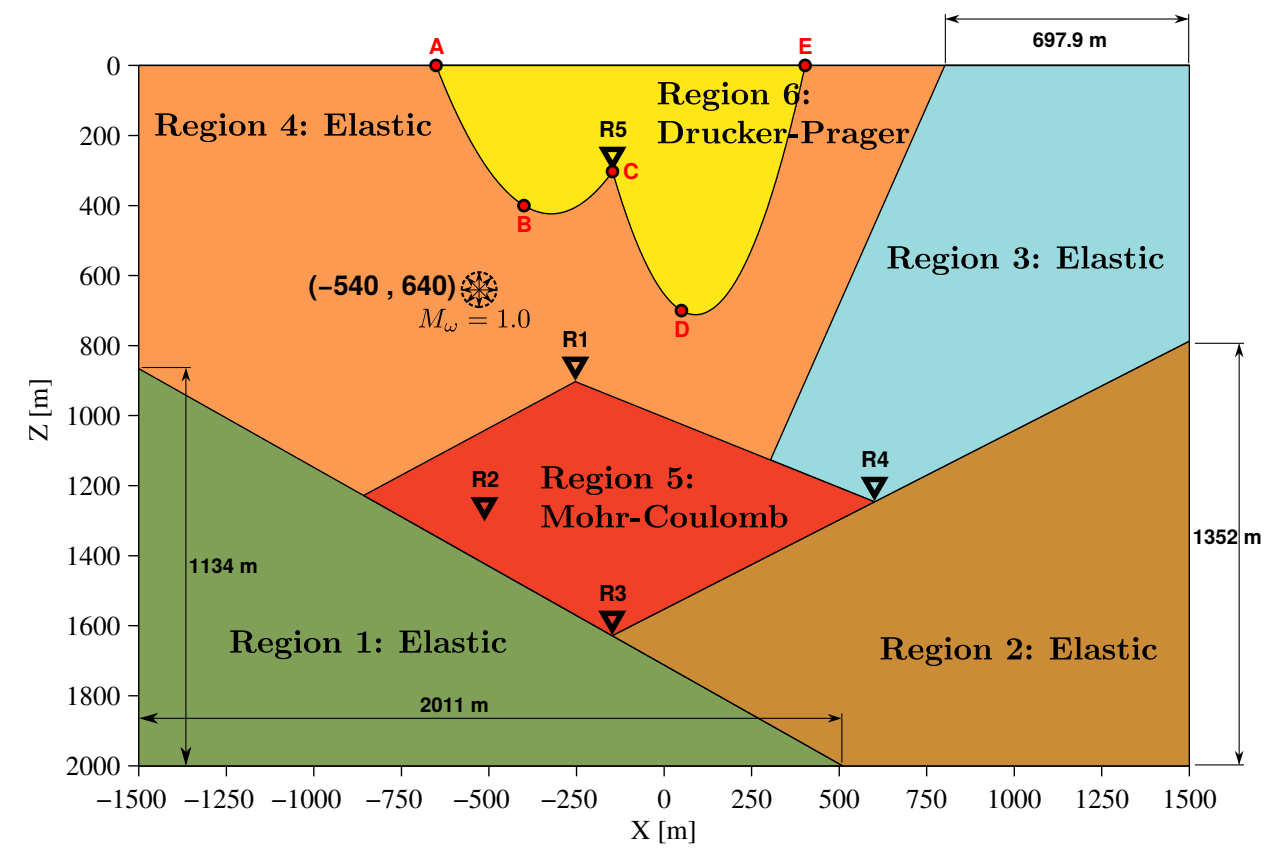

Figure 7: Geological setting. The five receivers are located at R1(-253,903), R2(-512.5, 1304.12), R3(-148, 1628.39), R4(601, 1245.99) and $\mathbf{R 5}(-147,303)$. The regions $1,2,3$ and 4 correspond to elastic materials, while the regions 5 and 6 have an elastoplastic behavior. The region 6 is made with two parabolic functions using the points $\mathbf{A}(-651,0), \mathbf{B}(-400,400), \mathbf{C}(-147,303), \mathbf{D}(50,700)$ and $\mathbf{E}(403,0)$. The first parabolic function is defined with the points $\mathbf{A}, \mathbf{B}$ and $\mathbf{C}$, while the second one is defined with $\mathbf{C}, \mathbf{D}$ and $\mathbf{E}$.

\begin{tabular}{|c|c|c|c|c|c|c|}
\hline Propierties $\rightarrow$ & $V p(\mathrm{~m} / \mathrm{s})$ & $V s(\mathrm{~m} / \mathrm{s})$ & $\rho\left(\mathrm{Kg} / \mathrm{m}^{3}\right)$ & $c(\mathrm{Kpa})$ & $\phi\left(^{\circ}\right)$ & $\psi\left({ }^{\circ}\right)$ \\
\cline { 1 - 6 } Region $\downarrow$ & & & & & & \\
\hline Region 1 & 2886 & 1666 & 1900 & - & - & - \\
\hline Region 2 & 1900 & 1096 & 1675 & - & - & - \\
\hline Region 3 & 3464 & 2000 & 1800 & - & - & - \\
\hline Region 4 & 1732 & 1000 & 1550 & - & - & - \\
\hline Region 5 & 2165 & 1250 & 1750 & 230 & 30 & 10 \\
\hline Region 6 & 1299 & 750 & 1600 & 170 & 25 & 15 \\
\hline
\end{tabular}

Table 3: Material properties of the geological setting.

permanent deformations, and maximum amplitudes at every station. Similarly, the oscillatory behavior of the velocity field is well tracked by the SCM approach.

Moreover, an overview for the amplitudes of the displacement field is shown in fig. 10 . At $t=0.768 \mathrm{~s}$, one can sees on the top left interface of the region 5 that the soil is permanently deformed. This situation also takes place inside region 6 near the free surface at $t=1.265 \mathrm{~s}$. These events are consistents with the plastic zones in fig. 8. In conclusion, the propagation phenomenon is correctly simulated by the SCM in comparison with the reference solution.

Finally, we examine the absolute error of the total strain energy at regions 5 and 6 . The energy $E^{i}$ is a time function and it is calculated with the total strain and stress tensors as follow:

$$
E_{i}=\frac{1}{2} \int_{\Omega_{i}} \varepsilon: \sigma d \Omega_{i},
$$

As we can observe in fig. 11, the strain energy of region 5 shows a peak at $t \approx 0.68 \mathrm{~s}$ when the incoming wave is refracted inside this region. The second peak (at $t \approx 2.5 \mathrm{~s}$ ) with less energy, is the result of the reflected waves by the ondulated basin and the free surface. In region 6 , the strain energy shows an exponential decay 

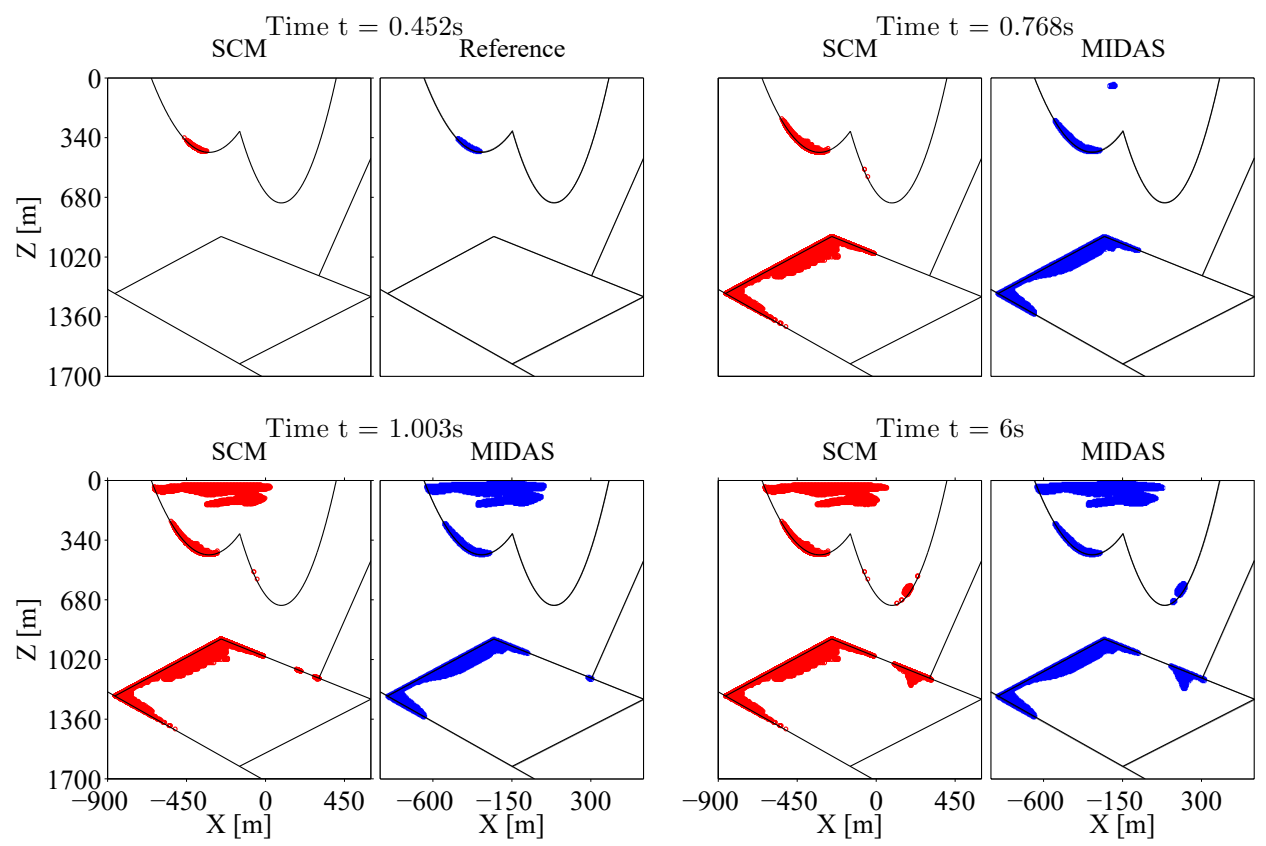

Figure 8: Evolution of the plastic activitys. The figures with blue points correspond to the reference solution, while the figures with red points belong to SCM solution.

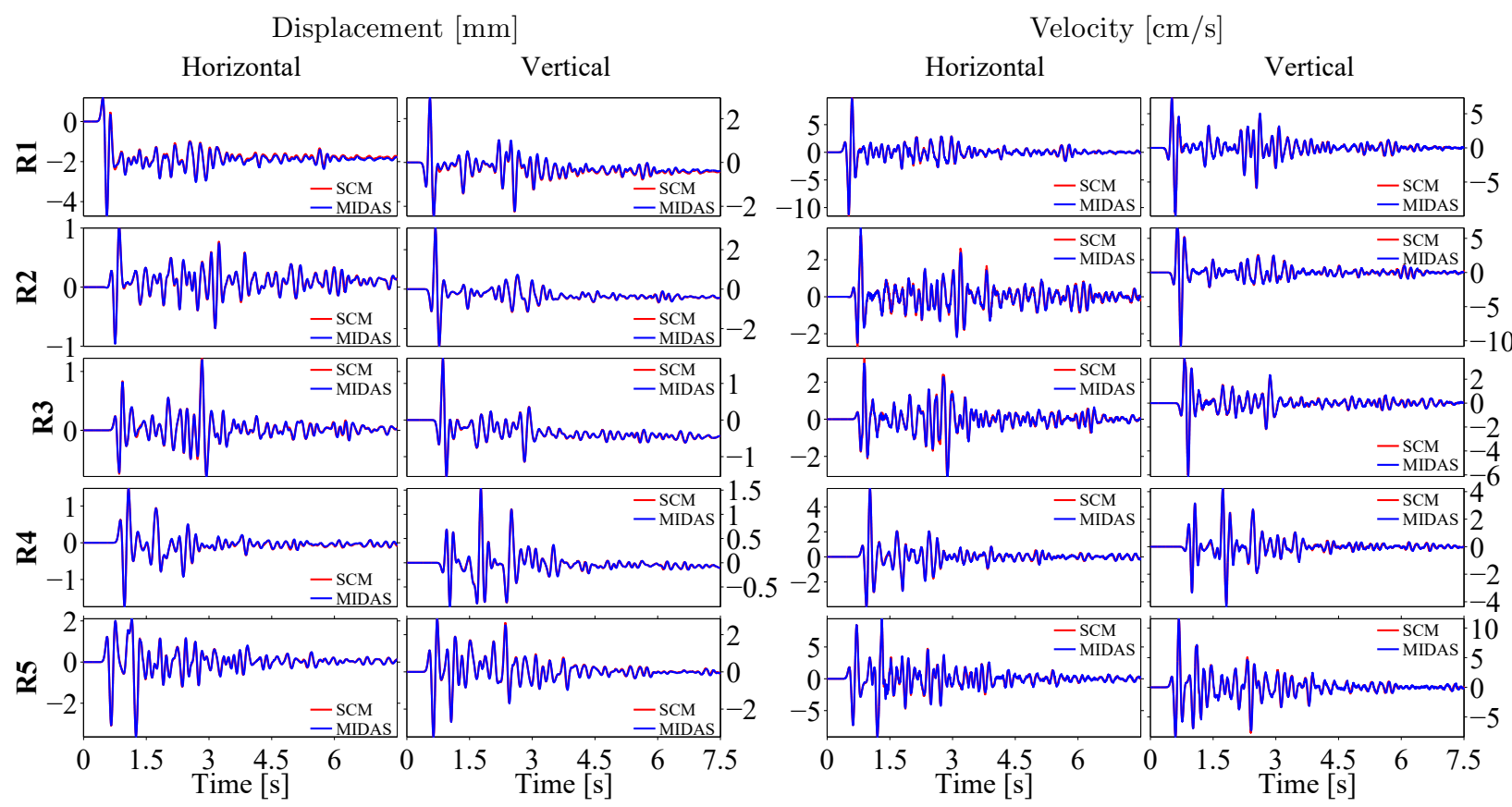

Figure 9: Comparison of the horizontal and vertical displacement $(\mathrm{mm})$ and velocity $(\mathrm{cm} / \mathrm{s})$ of the five receivers using 1/12 combination. The colum one and two are the horizontal and vertical displacements respectively. The column three is the horizontal velocity and the last one is the vertical velocity. The red line in each graph is the solution obtained by the SCM, while the blue line is the reference solution. 
from $t \approx 0.75 \mathrm{~s}$, because of the incident wave travels through the basin and then is slowly refracted downward into the bedrock. Nevertheless, the SCM shows that it is capable to predict the history of the strain energy with high accuracy. However, the absolute error obtained by comparision with the reference results presents a small difference when we used $P=1$ and $P=3$. Nevertheless, the CPU time (see table 4) for $P=3$ is lower than its counterpart $P=1$. In addition, when $P P W$ increases, the absolute error does not decrease significantly.

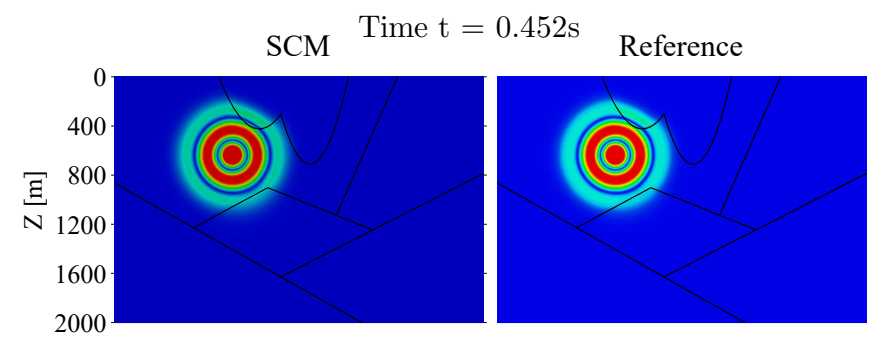

Time $\mathrm{t}=1.003 \mathrm{~s}$
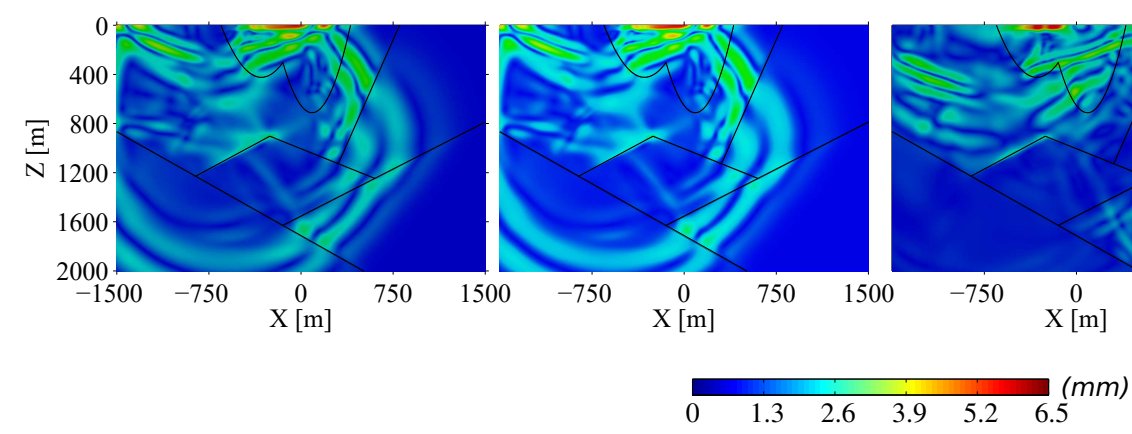

SCM Time $\mathrm{t}=0.768 \mathrm{~s} \quad$ Reference
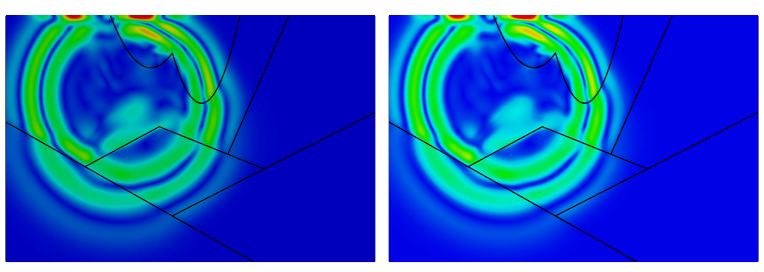

Time $\mathrm{t}=1.265 \mathrm{~s}$

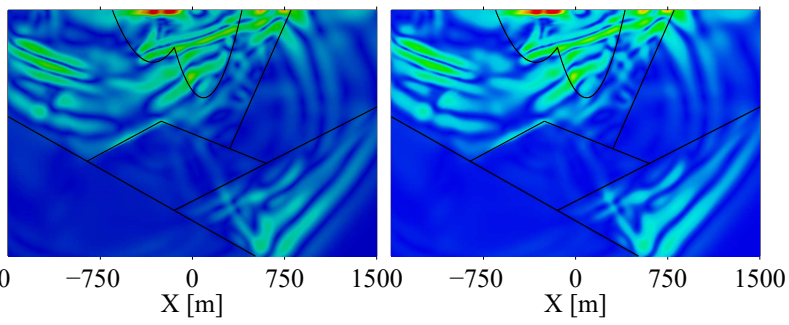

Figure 10: Snapshots of the displacement norm in different time steps using the combination $P / P P W=3 / 12$ for the SCM.

\begin{tabular}{|c|c|c|c|c|c|c|}
\hline $\begin{array}{c}\text { Points per } \\
\text { Wavelength } \\
\begin{array}{c}\text { Polynomial } \\
\text { Degree }\end{array}\end{array}$ & \multicolumn{3}{|c|}{$P P W=12$} & \multicolumn{3}{c|}{$P P W=16$} \\
\hline$P=1$ & $\begin{array}{c}\text { CPU Time } \\
23 \text { hours } \\
8 \text { mins }\end{array}$ & 154401 & $5.41 \times 10^{-4}$ & $\begin{array}{c}35 \text { hours } \\
18 \text { mins }\end{array}$ & 265651 & $4.12 \times 10^{-4}$ \\
\hline$P=3$ & $\begin{array}{c}17 \text { hours } \\
20 \text { mins }\end{array}$ & 152482 & $4.52 \times 10^{-4}$ & $\begin{array}{c}30 \text { hours } \\
33 \text { mins }\end{array}$ & 265651 & $3.42 \times 10^{-4}$ \\
\hline
\end{tabular}

Table 4: CPU time, degrees of freedom and sampling time of all schemes in the irregular geological setting simulation. 

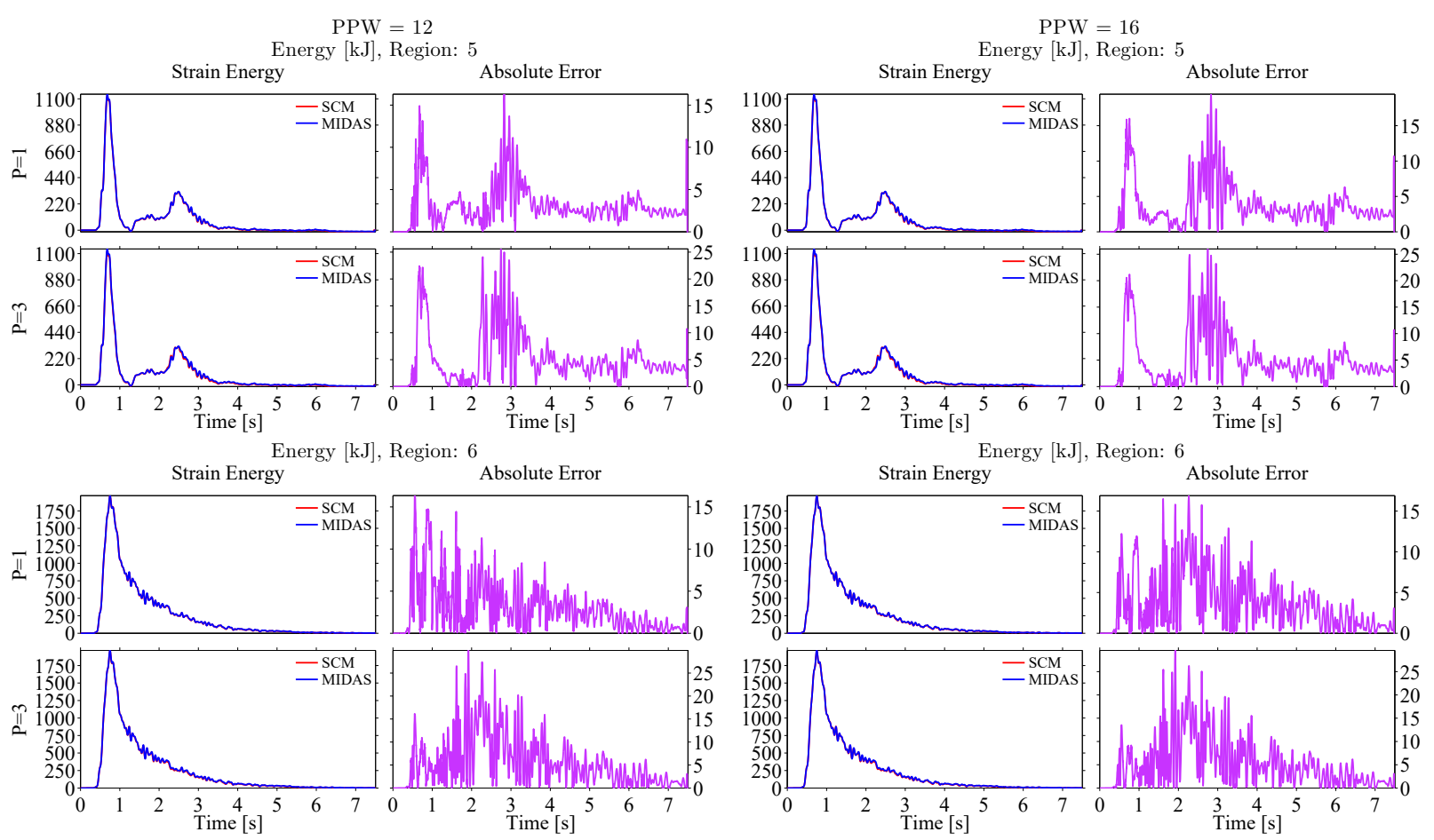

Figure 11: Strain energy and absolute error of regions 5 and 6 for combinations $P / P P W=1 / 12$ and $3 / 12$ for columns 1 , 2. And $1 / 16$ and $3 / 16$ for columns 3,4 .

\section{Concluding Remarks}

One of the main advantages of the spectral cell method is the fictitious-domain approach, which allowed us to reduce the meshing processing to a simple cartesian grid. The main feature of this kind of meshes is that the elemental mass and stiffness matrices of homogeneus elements are constants and depend only of the material parameters. In particular, the most important findings of our implementation are:

(i) Despite that the error results do not differ too much from $P P W=12$ to $P P W=16$ for both numerical experiments, a value of points per wavelength $P P W=12$ is enough to capture the permanent deformation and maximum amplitudes of both displacement and velocity fields. A value of $P P W=16$ has similar results, but it has higher computational cost.

(ii) When the polynomial degree increases from $P=1$ to $P=3$, the CPU time decreases $20 \%$ approximately, whereas the error for both polynomial degrees do not differ significantly. On the other hand, if we increase from $P=3$ to $P=5$, it is obtained a close agreement, but the CPU time increases twice approximately. In conclusion, the optimal value for the polynomial degree is $P=3$.

(iii) The analytical integration scheme together with the ideas of the adaptive integration based on the quadtree decomposition, are capable to track the sharp interfaces and re-entrant corners for the discontinuities of the materials inside the elements.

(iv) The SCM approach is capable to predict the total strain energy, even when a non-linear behavior is taking into account.

These findings allows us to conclude that the spectral cell method is a good candidate to simulate seismic events in complex geological structures that exhibit non-linear material behavior. 


\section{References}

Abedian, A., Parvizian, J., Düster, A., and Rank, E. (2013). The finite cell method for the J2 flow theory of plasticity. Finite Elements in Analysis and Design, 69:37-47.

Ainsworth, M. and Wajid, H. A. (2009). Dispersive and dissipative behavior of the spectral element method. SIAM Journal on Numerical Analysis, 47(5):3910-3937.

Bao, H., Bielak, J., Ghattas, O., Kallivokas, L. F., O'Hallaron, D. R., Shewchuk, J. R., and Xu, J. (1998). Largescale simulation of elastic wave propagation in heterogeneous media on parallel computers. Computer Methods in Applied Mechanics and Engineering, 152:85-102.

Bielak, J., Ghattas, O., and Kim, E. J. (2005). Parallel octree-based finite element method for large-scale earthquake ground motion simulation. Computer Modeling in Engineering and Sciences, 10(2):99-112.

Casarotti, E., Stupazzini, M., Lee, S. J., Komatitsch, D., Piersanti, A., and Tromp, J. (2008). Cubit and seismic wave propagation based upon the spectral-element method: An advanced unstructured mesher for complex 3d geological media. In Proceedings of the 16th International Meshing Roundtable, pages 579-597. Springer.

Cook, R. D., Malkus, D. S., and Plesha, M. E. (1989). Concepts and applications of finite element analysis. John Wiley \& Sons.

Cupillard, P., Delavaud, E., Burgos, G., Festa, G., Vilotte, J.-P., Capdeville, Y., and Montagner, J.-P. (2012). Regsem: a versatile code based on the spectral element method to compute seismic wave propagation at the regional scale. Geophysical Journal International, 188(3):1203-1220.

de Souza Neto, E. A., Perić, D., and Owen, D. R. J. (2008). Computational Methods for Plasticity. John Wiley and Sons, Ltd.

Drucker, D. C. and Prager, W. (1952). Soil mechanics and plastic analysis or limit design. Quarterly of Applied Mathematics, 10(2):157-165.

Duczek, S., Joulaian, M., Düster, A., and Gabbert, U. (2014a). Numerical analysis of Lamb waves using the finite and spectral cell methods. International Journal for Numerical Methods in Engineering, 99(1):26-53.

Duczek, S., Liefold, S., and Gabbert, U. (2014b). The finite and spectral cell methods for smart structure applications: Transient analysis. Acta Mechanica, pages 1-25.

Duczek, S., Liefold, S., and Gabbert, U. (2014c). The Spectral Cell Method for Ultrasonic Guided Wave Propagation Problems. In EWSHM-7th European Workshop on Structural Health Monitoring.

Düster, A., Parvizian, J., Yang, Z., and Rank, E. (2008). The finite cell method for three-dimensional problems of solid mechanics. Computer Methods in Applied Mechanics and Engineering, 197(45):3768-3782.

Field, E., Kramer, S., Elgamal, A.-W., Bray, J., Matasovic, N., Johnson, P., Cramer, C., Roblee, C., Wald, D., Bonilla, L., et al. (1998). Nonlinear site response: where we're at (a report from a scec/peer seminar and workshop). Seismological Research Letters, 69(3):230-234.

Frankel, A. (1993). Three-dimensional simulations of ground motions in the San Bernardino Valley, California, for hypothetical earthquakes on the San Andreas fault. Bulletin of the Seismological Society of America, 83(4):1020-1041.

Gelagoti, F., Kourkoulis, R., Anastasopoulos, I., and Gazetas, G. (2012). Nonlinear dimensional analysis of trapezoidal valleys subjected to vertically propagating SV waves. Bulletin of the Seismological Society of America, 102(3):999-1017.

Graves, R. W. and Pitarka, A. (2010). Broadband ground-motion simulation using a hybrid approach. Bulletin of the Seismological Society of America, 100(5A):2095-2123. 
Hinton, E., Rock, T., and Zienkiewicz, O. (1976). A note on mass lumping and related processes in the finite element method. Earthquake Engineering \& Structural Dynamics, 4(3):245-249.

Hoek, E. and Brown, E. T. (1980). Empirical strength criterion for rock masses. Journal of the Geotechnical Engineering Division, 106(GT9):1013-1035.

Joulaian, M., Duczek, S., Gabbert, U., and Düster, A. (2014). Finite and spectral cell method for wave propagation in heterogeneous materials. Computational Mechanics, 54(3):661-675.

Komatitsch, D. and Tromp, J. (2002a). Spectral-element simulations of global seismic wave propagation-I. Validation. Geophys. J. Int., 149(2):390-412.

Komatitsch, D. and Tromp, J. (2002b). Spectral-element simulations of global seismic wave propagation-II. threedimensional models, oceans, rotation and self-gravitation. Geophysical Journal International, 150(1):303-318.

Komatitsch, D. and Vilotte, J.-P. (1998). The spectral element method: An efficient tool to simulate the seismic response of 2D and 3D geological structures. Bulletin of the Seismological Society of America, 88(2):368-392.

Lysmer, J. and Kuhlemeyer, R. L. (1969). Finite dynamic model for infinite media. Journal of Engineering Mechanics, ASCE, 95(EM4):859-877.

Midas-GTS-NX (2016). version 1.1 (April 6). MIDAS Information Technology, Seongnam, South Korea. Available at http://midasgtsnx. com.

Moczo, P., Kristek, J., Galis, M., Pazak, P., and Balazovjech, M. (2007). The finite-difference and finite-element modeling of seismic wave propagation and earthquake motion. Acta Physica Slovaca., 57(2):177-406.

Olsen, K. B. and Archuleta, R. J. (1996). Three-dimensional simulation of earthquakes on the Los Angeles fault system. Bulletin of the Seismological Society of America, 86(3):575-596.

Parvizian, J., Düster, A., and Rank, E. (2007). Finite cell method. Computational Mechanics, 41(1):121-133.

Pelties, C., Käser, M., Hermann, V., and Castro, C. E. (2010). Regular versus irregular meshing for complicated models and their effect on synthetic seismograms. Geophysical Journal International, 183(2):1031-1051.

Restrepo, D., Taborda, R., and Bielak, J. (2011). Effects of soil nonlinearity on ground response in 3D simulations - An application to the Salt Lake City basin. Proceedings of the 4th IASPEI/IAEE International Symposium: Effects of Surface Geology on Seismic Motion, University of California, Santa Barbara, August 23-26.

Roscoe, K. H. and Burland, J. (1968). On the generalized stress-strain behaviour of wet clay. In Heyman, J. and Leckie, F. A., editors, Engineering Plasticity, pages 535-609. Cambridge University Press.

Ruess, M., Tal, D., Trabelsi, N., Yosibash, Z., and Rank, E. (2012). The finite cell method for bone simulations: verification and validation. Biomechanics and modeling in mechanobiology, 11(3-4):425-437.

Saul'ev, V. K. (1963). On solution of some boundary value problems on high performance computers by fictitious domain method. Siberian Math. J, 4(4):912-925 (in Russian).

Schillinger, D., Ruess, M., Zander, N., Bazilevs, Y., Düster, A., and Rank, E. (2012). Small and large deformation analysis with the $p$-and $b$-spline versions of the finite cell method. Computational Mechanics, 50(4):445-478.

Seed, H. B. and Idriss, I. M. (1969). Influence of soil conditions on ground motions during earthquakes. Journal of the Soil Mechanics and Foundations Division, 95(1):99-138.

Sekiguchi, H. and Ohta, H. (1977). Induced anisotropy and time dependency in clays. $9^{\text {th }}$ ICSMFE, pages 229-238.

Seriani, G. and Priolo, E. (1994). Spectral element method for acoustic wave simulation in heterogeneous media. Finite Elements in Analysis and Design, 16(3-4):337-348. 
Simo, J. C. and Hughes, T. J. R. (1998). In Marsden, J. E., Wiggins, S., and Sirovich, L., editors, Computational Inelasticity, volume 7 of Interdisciplinary Applied Mathematics. Springer New York.

Stupazzini, M., Paolucci, R., and Igel, H. (2009). Near-fault earthquake ground-motion simulation in the grenoble valley by a high-performance spectral element code. Bulletin of the Seismological Society of America, 99(1):286-301.

Szabo, B. A. and Babuška, I. (1991). Finite element analysis. John Wiley \& Sons.

Taborda, R. and Bielak, J. (2013). Ground-motion simulation and validation of the 2008 Chino Hills, California, Earthquakes. Bulletin of the Seismological Society of America, 103(1):131-156.

Taborda, R., Bielak, J., and Restrepo, D. (2012). Earthquake ground-motion simulation including nonlinear soil effects under idealized conditions with application to two case studies. Seismological Research Letters, 83(6):1047-1060.

Xu, J., Bielak, J., Ghattas, O., and Wang, J. (2003). Three-dimensional nonlinear seismic ground motion modeling in basins. Physics of the Earth and Planetary Interiors, 137:81 - 95.

Zander, N., Kollmannsberger, S., Ruess, M., Yosibash, Z., and Rank, E. (2012). The finite cell method for linear thermoelasticity. Computers \& Mathematics with Applications, 64(11):3527-3541. 